\title{
SPRACHFÖRDERUNG DURCH EXTENSIVES LESEN UND HÖREN IM UNIVERSITÄREN DAF-UNTERRICHT
}

\begin{abstract}
In diesem Beitrag werden Ergebnisse einer empirischen Studie dargestellt, die 2014/15 an der Philosophischen Fakultät in Novi Sad (Serbien) mit Studierenden des Wahlfachs Deutsch (Niveaustufe A2) durchgeführt wurde. Während in der Experimentalgruppe das extensive Verfahren angewandt wurde, wurde der Kontrollgruppe regulärer Unterricht erteilt, im Rahmen dessen verschiedene Lehrwerke und eine Vielfalt an didaktischen Materialien eingesetzt wurden. Die Dauer der Untersuchung betrug zwei Semester. Im Anschluss wurden die Lernenden in Hinblick auf ihre Sprachkompetenz getestet. Die Daten wurden quantitativ ausgewertet, während die Gruppenunterschiede mithilfe des t-Tests statistisch untersucht wurden. Die Ergebnisse der Untersuchungen weisen darauf hin, dass sich extensive Lese- und Hörprogramme vor allem auf die produktiven Sprachkompetenzen positiv auswirken.
\end{abstract}

Schlüsselwörter: extensives Lesen und Hören, experimentelle Untersuchung, authentische Texte, DaF-Unterricht.

\section{FACILITATION OF LANGUAGE USE THROUGH EXTENSIVE READING AND LISTENING IN FOREIGN LANGUAGE TEACHING IN HIGHER EDUCATION}

ABSTRACT: This paper presents the results of an empirical study conducted in $2014 / 15$ at the Faculty of Philosophy in Novi Sad (Serbia) with students who have chosen German as an elective course (level A2). While the extensive approach was implemented in the experimental group, the control group was given regular instruction using various textbooks and a variety of didactic materials. The duration of the study was two semesters, after which the learners' language skills were tested. The quantitative data has been analysed accompanied by a statistical analysis of group differences using the t-test. The results of the study indicate a positive impact of extensive reading and listening programs on productive language proficiency.

Key words: extensive reading and listening, empirical investigation, authentic texts, teaching German as a foreign language. 


\section{EINFÜHRUNG}

Die deutsche Sprache hat in Serbien eine lange Tradition und wird an Bildungseinrichtungen jeder Art gelernt. Das Problem ist aber, dass der Input zum Deutschlernen zu gering ist und sich nur auf das Lehrwerk und Zusatzmaterialien beschränkt (Übungshefte und Grammatiken). Die Lernenden haben nur im Unterricht Kontakt zur deutschen Sprache, während sie außerhalb des Unterrichts die eigene Muttersprache verwenden. Auch im häuslichen Umfeld fehlt der mediale Kontakt zur deutschen Sprache (Stipančević 2013; Stipančević 2015). In der schulischen und universitären Lernumgebung wird die Sprache vor allem bewusst gelernt, was den gesamten Spracherwerbsprozess verlangsamt und erschwert. Um die Effizienz des Fremdsprachenlernens zu erhöhen, ist vor allem der massive Kontakt zur Zielsprache von Bedeutung. Werden die Lernenden der Fremdsprache in einem größeren Umfang ausgesetzt, werden nicht nur bewusste sondern auch unbewusste Lernprozesse in Gang gesetzt (Koeppel 2013), die ein effizienteres Lernen ermöglichen.

Der extensive Ansatz baut auf der Input- und Output-Hypothese sowie auf der konstruktivistischen Lerntheorie auf. Im Rahmen dieser drei Lerntheorien werden folgende Elemente als wichtige Voraussetzung für erfolgreiche Spracherwerbsprozesse herausgestellt (vgl. Decke-Cornill \& Küster 2014; Krashen 1985; Wolff 1997):

1. Massiver Kontakt mit der Fremdsprache;

2. Anwendung von Sprachkenntnissen durch Sprechen und Schreiben;

3. Reiche, vielfältige, offene und herausfordernde Lernumgebung;

4. Individualisierung und Differenzierung im Unterricht.

Ein herkömmlicher Unterricht, der nur mit einem Lehrwerk erfolgt, kann diesen Forderungen nicht gerecht werden, sondern verlangt nach Ergänzungen in Form von digitalen Medien und Methoden, die eine Individualisierung und Differenzierung ermöglichen, und den Lernenden in den Vordergrund des Lernprozesses stellen. So eine Methode stellt der extensive Ansatz dar, der auch die Integration digitaler Medien in den Lernprozess ermöglicht und den Einbezug von Lese- und Hörtexten miteinschließt.

\section{DAS EXTENSIVE LESEN}

Unter „extensivem Lesen“ versteht man das Lesen von längeren Texten in möglichst hoher Frequenz, wobei die Lernenden selbst bestimmen, welche Texte 
sie lesen werden (Biebricher 2008: 39). Aus diesem Grund wird dieser Ansatz auch als reading for pleasure bezeichnet (Day \& Bamford 1998). Eines der Ziele des extensiven Lesens ist es, die Lesemotivation zu steigern (Kirchhoff 2009), aber auch die Lesekompetenz durch die Erfahrung der Sprache im Kontext zu verbessern (Edmondson 1996; Elley 1991; Elley \& Mangubhai 1981). Für diese Leseart ist zudem charakteristisch, dass Texte auf globaler Ebene verstanden werden (Biebricher 2008: 39), was einem Rezeptionsverfahren in der Muttersprache gleichkommt. Auf diese Art soll der Lesende Spaß am Lesen entwickeln, spontan seine Lesestrategien verbessern und auch unbewusst seinen Wortschatz erweitern. Für extensive Leseprogramme ist außerdem charakteristisch, dass das Lesen individualisiert und still erfolgt, damit jeder Leser in seinem eigenen Tempo lesen kann. Die Texte werden hierbei normalerweise nicht im Unterricht bzw. Plenum besprochen (Barnett 1989: 167). Die eigenen Erfahrungen aus der Praxis zeigen aber, dass gerade die Besprechung der Texte im Unterricht sehr fruchtbar sein kann ist. Einerseits wird dadurch die Sprechkompetenz kontinuierlich gefördert, andererseits bringt die Behandlung verschiedener Themen viel Abwechslung und Lebendigkeit in den Unterrichtsprozess. Dabei müssen nicht alle Lernenden die Texte präsentieren, die sie gelesen haben. Es wird empfohlen, dass die Lerner sich zuerst in Partner- oder Gruppenarbeit miteinander austauschen und die Lehrperson daraufhin ein paar Lernende aufruft, ihre Beiträge zu präsentieren. Nach jedem Beitrag kann die Lehrperson zusätzliche Fragen stellen und so eine Diskussion in Gang setzten, in die auch die anderen Teilnehmer einbezogen werden können. Praktisch ist diese Vorgehensweise vor allem bei Unterrichtseinheiten, die 90 Minuten dauern. In jedem Fall ist die Verbindung des Lesens mit produktiven Aktivitäten (Sprechen und Schreiben) wichtig. Verschiedene Studien haben nämlich auch nachgewiesen, dass sich dies besonders förderlich auf die Spracherwerbsprozesse auswirkt (Hulstijn 1992; Nation 2001; Paribakht, Wesche 1997; Stipančević 2016).

Die Auswahl der Lesetexte ist einer der entscheidenden Faktoren für den Erfolg des extensiven Programms. Laut Biebricher (2008) müssen Texte verschiedener Schwierigkeitsstufen verwendet werden, sodass die Lerner weder über- noch unterfordert werden. $\mathrm{Da}$ in der Muttersprache häufig Unterhaltungsliteratur gelesen wird, wird empfohlen, dass auch solche Texte ins extensive Programm aufgenommen werden (Brusch 1994). Außerdem sollte man den Lernenden auch authentische Sachtexte anbieten wie Texte aus Zeitungen, Zeitschriften und Blogs, Berichte, Kommentare, Buchtipps und Nachrichten, da im Rahmen verschiedener Untersuchungen zum Thema ,Motivation“ authentische Texte von Lernenden als stark motivierend bewertet wurden (Düwell 1979; 
Stipančević 2015, 2016; Storch 2009). Auch wenn einige authentische Texte auf den ersten Blick schwierig wirken, wird sich der Lernende, falls Motivation und Interesse an Text und Thema bestehen, besonders anstrengen, die Texte zu verstehen.

Bei den verschiedenen extensiven Leseprogrammen besteht Uneinigkeit über den Umfang des extensiven Lesens. Die Angaben dazu sind unterschiedlich und variieren von 500 Seiten pro Semester, bis hin zu 400 Wörtern täglich (Silva 1992), oder auch 20 Minuten täglich (Seow 1999). Diese Angaben beziehen sich vor allem auf das Lesen von Texten im Englischunterricht. Für den DaF-Unterricht muss man noch differenzierter vorgehen und mit kürzeren Texten beginnen, da die Sprachkenntnisse der Deutschlernenden anfangs in der Regel geringer sind als die der Englischlernenden. Werden sofort lange Texte eingesetzt, läuft man Gefahr, die Lernenden zu demotivieren, statt sie zu motivieren. Mit der Zeit können dann auch längere Texte eingeführt werden.

Die Aufgabe der Lehrkraft ist es, die Lernenden auf verschiedene Internetseiten hinzuweisen, wo die Lernenden die Texte dann selbst auswählen, da der Faktor „Lesemotivation“ eine wichtige Rolle im Textverstehen spielt. Im Rahmen verschiedener Untersuchungen aus dem Bereich der Lesepsychologie wurde nämlich festgestellt, dass Texte leichter bzw. besser verstanden werden, je mehr sie den Interessen der Lernenden entsprechen (Estes \& Vaughan 1973).

\section{DAS EXTENSIVE HÖREN}

Das Hörverstehen gehört $\mathrm{zu}$ den wichtigsten Kompetenzen im Fremdsprachenunterricht. Das Hören nimmt mit 55\% eine besondere Stellung in der muttersprachlichen Kommunikation ein, während das Sprechen mit 23\% in der alltäglichen Kommunikation an zweiter Stelle steht, das Lesen mit 13\% die dritte Stelle und das Schreiben mit 9\% die letzte Stelle besetzt. Dem Hörverstehen kommt in Bezug auf seinen Einfluss auf die Entwicklung der anderen Sprachkompetenzen eine wichtige Bedeutung zu. Hörverstehenstexte geben den sprachlichen Input, der für einen effektiven Spracherwerbsprozess von großer Bedeutung ist (Surkamp 2010). Auf der anderen Seite kommt das isolierte Hörverstehen in der Realität sehr selten vor, weil das gesprochene Wort sehr oft von visuellen Medien (Kino, Fernsehen, Video) begleitet wird, weshalb man in der Fremdsprachendidaktik auch vom Hörsehverstehen spricht (Storch 2009). Aus diesem Grund sollten im Fremdsprachenunterricht neben Audio- auch audiovisuelle Medien eingesetzt werden, die auf die Entwicklung des Hörsehverstehens abzielen. 
Unter dem Begriff „Extensives Hören“ versteht man das Hören einer größeren Anzahl von Texten, mit dem Ziel eines globalen Textverständnisses. Dabei werden die Texte wieder von den Lernenden ausgewählt, die sie hören werden. Das Ziel dieses Ansatzes ist die Schulung des Hörverstehens, aber auch die Entwicklung der produktiven Kompetenzen.

Positive Effekte des extensiven Hörens auf die Sprachkompetenzen wurden bereits in einigen Untersuchungen nachgewiesen, insbesondere im Spanischunterricht (Weyers 1999) und im DaF-Unterricht (Stipančević 2014). Hier wurde festgestellt, dass ein regelmäßiges Ansehen von Fernsehserien den Wortschatz der Lernenden vergrößert.

Zur erfolgreichen Umsetzung dieses Ansatzes mit Schwerpunkt auf dem Hör(seh)verstehen ist zudem wichtig, dass die Lernenden die Möglichkeit haben, die Inhalte selbstständig auszuwählen, die sie hören (sehen) werden. Dank dem Internet sind heutzutage viele deutsche Serien auch online verfügbar, z.B.: Alarm für Cobra Elf, Unter uns, Alles was zählt, Der Lehrer, Türkisch für Anfänger, Berlin Berlin, Dark, Ku'damm. Natürlich kann man Lernenden auch Filme, verschiedene Sendungen oder auch Podcasts anbieten. Die Hauptsache ist, dass diese Inhalte nicht zu kurz sind und beim Lernenden Interesse wecken.

Der Großteil extensiver Programme (mit Schwerpunkt auf dem Lesen) findet im asiatischen Raum mit Erwachsenen statt (Biebricher 2008: 41). An deutschen Schulen gibt es Vorschläge zu „Bücherkisten“ im Fremdsprachenunterricht der Grundschule (Börner \& Brusch 2004; BurwitzMelzer 2004) und seit den 90-er Jahren wird auch die Gründung englischer Klassenbibliotheken in der Sekundarstufe I angeregt (Biederstädt 1990; Stenzel 1990; 1994). Allerdings wird der Schule vor allem die Aufgabe zugedacht, Lesestrategien zu vermitteln, während von den Lernenden erwartet wird, dass sie in ihrer Freizeit Texte in der Fremdsprache lesen (Ross \& Porteous-Schwier 2005; Volkmann 2000). Es reicht aber nicht den Lernenden zu sagen, dass sie zu Hause viel lesen und fernsehen sollen. Man muss ihnen den Weg zeigen, mit ihnen einmal das Programm durchgehen, ihr Interesse am Lesen und Hören von Texten und Sendungen auf Deutsch wecken, bis es für sie zur Gewohnheit wird. Im folgenden Abschnitt dieses Artikels wird aufgezeigt, wie der extensive Ansatz mit Lernenden im DaF-Unterricht eingesetzt wurde und welche Effekte dies auf ihre Sprachkompetenzen hatte. 


\section{EXTENSIVER ANSATZ IM UNIVERSITÄREN REMDSPRACHENUNTERRICHT}

Die experimentelle Untersuchung, die Effekte des extensiven Lesens und Hörens untersuchte, wurde an der Philosophischen Fakultät in Novi Sad mit Studierenden im Wahlfach Deutsch durchgeführt. Die Untersuchung umfasste 34 Studierende der Studiengänge Englische Sprache und Literatur, Pädagogik, Psychologie, Russische Sprache und Literatur, Slowakische Sprache und Literatur, Soziologie, Journalistik, Philosophie, Ungarische Sprache und Literatur, Geschichte, Vergleichende Literaturwissenschaft, Rumänische Sprache und Literatur sowie Serbische Sprache und Literatur. Im Rahmen all dieser Studiengänge wird Deutsch als Wahlfach angeboten.

Die Studierenden wurden nach einem Einstufungstest in zwei Gruppen eingeteilt: eine Gruppe war hierbei die Experimentalgruppe, mit der das extensive Programm durchgeführt wurde, und die andere Gruppe war die Kontrollgruppe, der regulärer Unterricht erteilt wurde. Nach der Einteilung der Studierenden in Gruppen, wurden noch drei Pretests durchgeführt, die gezeigt haben, dass keine statistisch signifikanten Unterschiede in den Leistungen der Gruppen bestanden.

Die Lernenden waren auf der Niveaustufe A2. Der Unterricht fand zweimal pro Woche statt und die Unterrichtsstunden dauerten jeweils 90 Minuten. Die gesamte Untersuchung dauerte zwei Semester (insgesamt 48 Unterrichtsstunden), von Oktober 2014 bis Juni 2015.

\subsection{Unterricht in der Kontrollgruppe}

In der Kontrollgruppe wurde ein regulärer Unterricht durchgeführt, in dem verschiedene Lehrwerke eingesetzt wurden: Schritte International (Hilpert et al. 2006, 2007), Optimal (Müller et al. 2011), Delfin (Aufderstraße et al. 2003). Auch Zusatzmaterialien wie Grammatik und Konversation (Swerlova 2013), über die Grammatik in einer kommunikativen Situation geübt werden soll, kamen zur Anwendung. In der Kontrollgruppe wurden auch Medien wie Computer, Beamer und Power-Point-Präsentationen sowie Methoden verwendet, die den Lernenden in den Vordergrund stellen und die Aktivität und Beteiligung der Lernenden fördern. Alle vier Sprachkompetenzen wurden gleichmäßig gefördert, sodass die Lernenden am Ende des Kurses imstande waren, ihre Sprachkenntnisse in der schriftlichen und mündlichen Kommunikation anzuwenden. Der Unterricht in der Kontrollgruppe war also keinesfalls traditionell, sondern wurde nach der 
kommunikativen Methode und nach den Prinzipien des modernen Fremdsprachenunterrichts organisiert und gestaltet.

Da Hausaufgaben in dieser Untersuchung eine wichtige Rolle spielen, wird auch auf die Aufgaben der Gruppen im Rahmen der Hausarbeit eingegangen. In der Kontrollgruppe bestanden die Hausaufgaben darin, dass die Lernenden eine Wortschatz- oder eine Grammatikaufgabe lösten, Aufsätze schrieben, einen Text im Lehrbuch lasen und Fragen dazu beantworteten. Dies sind typische Hausaufgabenformate, die Lehrende ihren Lernenden üblicherweise aufgeben.

\subsection{Unterricht in der Experimentalgruppe}

Die gleichen Themen, die in der Kontrollgruppe mithilfe eines Lehrwerks bearbeitet wurden, wurden in der Experimentalgruppe mithilfe von authentischen Texten durchgenommen. Neben Drucktexten, wurden auch mehrmals Lieder, Filme und Fernsehserien in den Unterricht eingebaut. Zugleich wurde aber auch nicht ganz auf ein Lehrwerk (Delfin) verzichtet, da es sich um das Sprachniveau A2 handelte. Es wurde verwendet, um den thematischen Wortschatz vorzustellen sowie Dialog- und Grammatikübungen durchzuführen (ungefähr 5\%).

Das extensive Programm wurde im Rahmen der Hausarbeit durchgeführt. Die Lernenden sollten nach jeder Stunde entweder authentische Drucktexte lesen (Zeitungsartikel, Berichte, Lesetipps und andere Arten von Sachtexten) oder Filme/Fernsehserien/Sendungen auf Deutsch ansehen. Es waren also sowohl mündliche als auch schriftliche Texte vertreten. Die Lernenden bestimmten selbst, welche Texte sie lesen bzw. welche Serie/Sendungen/Filme sie sich ansehen würden, um ihre Motivation zu steigern. Ein Minimalpensum bei Hörtexten waren 30 Minuten Hörzeit pro Woche. Auf diese Art und Weise wurde eine reichhaltige Lernumgebung geschaffen, was sich, laut konstruktivistischer Lerntheorie, positiv auf den Lernprozess auswirken soll.

Gemäß der Output-Hypothese sollte der Input mit produktiven Aktivitäten verbunden werden, da diese den Spracherwerbsprozess besonders vorantreiben. Aus diesem Grund haben die Lernenden im Rahmen dieser Untersuchung alle Texte, die sie gelesen und gehört haben, mit eigenen Worten zusammengefasst, ihre Meinung dazu geäußert und anschließend mündlich einstudiert, wodurch sowohl das Sprechen als auch das Schreiben geübt wurde. Im Unterricht wurden die Texte dann zunächst im Rahmen der Partnerarbeit und später im Plenum präsentiert, was zahlreiche Impulse zur weiteren Diskussion lieferte. Die Lernenden bewerteten diese Phasen der Unterrichtsstunde als besonders fruchtbar und interessant, da über aktuelle Themen und Ereignisse gesprochen und diskutiert 
wurde. Diese Phasen nahmen 20-30 Minuten der Unterrichtszeit ein, während in den übrigen 60-70 Minuten regulärer Unterricht erteilt wurde. Nach der Stunde wurden alle Aufgaben bei der Lehrperson abgegeben, die sie dann alle kontrollierte und ihre grammatische Richtigkeit prüfte. Den Lernenden wurde in der darauffolgenden Stunde eine Rückmeldung zu ihren Leistungen gegeben.

\section{ERGEBNISSE DER UNTERSUCHUNG}

Im Juni wurden die Lernenden erneut in Hinblick auf ihre Sprachkompetenzen getestet, und zwar mithilfe von 3 verschiedenen Tests. Im Folgenden werden zunächst die Ergebnisse der drei Pretests vorgestellt und anschließend die Ergebnisse der drei Posttests.

\subsection{Ergebnisse der Pretests}

Nachdem die Lernenden nach einem Einstufungstest in Gruppen eingeteilt wurden, wurden drei Pretests durchgeführt. Das Ziel war es, festzustellen, ob statistische Unterschiede in den Leistungen der Gruppen bestehen. Die Lernenden füllten zunächst einen standardisierten (Test 1) und danach zwei nicht standardisierte Tests aus, die von der Autorin selbst entwickelt wurden (Test 2 und $3)$.

Test 1

Der erste mit den Lernenden durchgeführte Test war ein standardisierter Test auf Niveau A2 (Gerbes \& Werff 2007). Im Rahmen dieses Tests wurden drei Kompetenzen getestet (Hörverstehen, Leseverstehen und Schreiben). In Tabelle 1 sind Ergebnisse von Test 1 dargestellt.

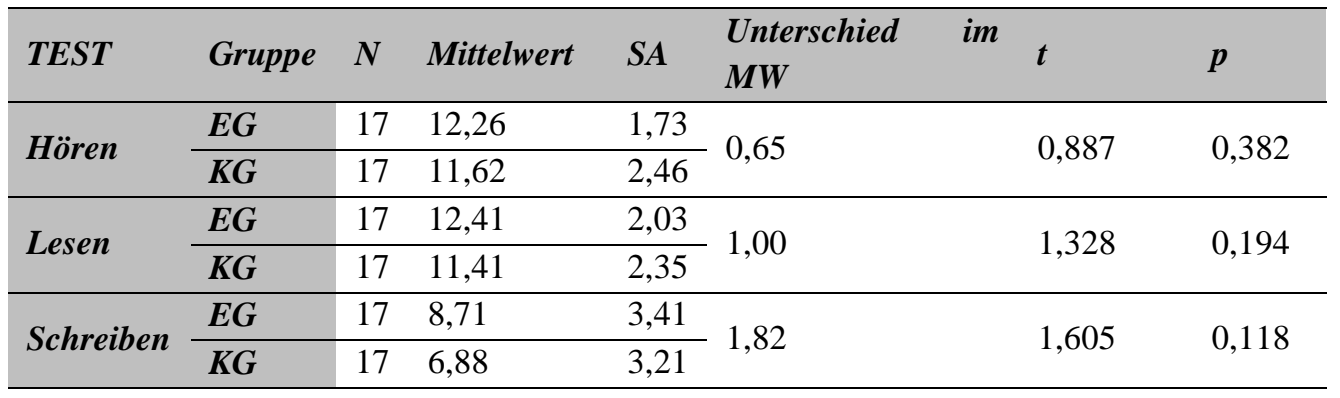

Tab. 1. Ergebnisse von Test 1 vor Durchführung der Untersuchung 
Die Ergebnisse von Test 1 zeigen, dass keine statistisch signifikanten Unterschiede in den Leistungen der Gruppen festgestellt wurden, da $p>0,05$ ist. Test 2

Test 2 war ein nicht standardisierter Test, der die Lesekompetenz anhand eines authentischen Textes prüfte (SpiegelOnline 2014). Der Text wurde leicht gekürzt und sprachlich vereinfacht, um eine Überforderung der Lernenden zu verhindern. Neben der Lesekompetenz wurde noch die Schreibkompetenz getestet, indem den Lernenden die Aufgaben gestellt wurden, ihre Meinung zum Text zu äußern und zwei Aufsätze zu schreiben. In Tabelle 2 werden die Testergebnisse dargestellt.

\begin{tabular}{|c|c|c|c|c|c|c|c|}
\hline TEST 2 & Gruppe & $N$ & $M W$ & $S A$ & Unterschied im MW & $t$ & $p$ \\
\hline \multirow{2}{*}{ Lesen } & $E G$ & 17 & 13,85 & 2,04 & \multirow{2}{*}{0,32} & \multirow{2}{*}{0,455} & \multirow{2}{*}{0,652} \\
\hline & $K G$ & 17 & 13,53 & 2,11 & & & \\
\hline \multirow{2}{*}{ Schreiben } & $E G$ & 17 & 3,82 & 3,51 & \multirow{2}{*}{$-0,53$} & \multirow{2}{*}{$-0,463$} & \multirow{2}{*}{0,647} \\
\hline & $K G$ & 17 & 4,35 & 3,15 & & & \\
\hline
\end{tabular}

Tab. 2. Ergebnisse von Test 2 vor Durchführung der Untersuchung

Die Ergebnisse des t-Tests zeigen, dass es keine statistisch signifikanten Unterschiede in den Leistungen der Gruppen gab, da der Wert $\mathrm{p}>0,05$ ist.

Test 3

Mit diesem Test wurde die Hörkompetenz anhand einer 25-minütigen Folge der Fernsehserie Mein Leben \& Ich geprüft. Auch die Schreibkompetenz wurde getestet, indem die Lernenden die gesehene Folge mit eigenen Worten zusammenfassen sollten.

\begin{tabular}{|c|c|c|c|c|c|c|c|}
\hline TEST 3 & Gruppe & $N$ & $M W$ & $S A$ & Unterschied im $M W$ & $t$ & $p$ \\
\hline \multirow{2}{*}{ Hören } & $E G$ & 17 & 7,74 & 2,11 & \multirow{2}{*}{0,53} & \multirow{2}{*}{0,679} & \multirow{2}{*}{0,502} \\
\hline & $K G$ & 17 & 7,21 & 2,43 & & & \\
\hline \multirow{2}{*}{ Schreiben } & $E G$ & 17 & 6,26 & 3,79 & \multirow{2}{*}{1,41} & \multirow{2}{*}{1,129} & \multirow[b]{2}{*}{0,267} \\
\hline & $K G$ & 17 & 4,85 & 3,49 & & & \\
\hline
\end{tabular}

Tab. 3. Ergebnisse von Test 3 vor Durchführung der Untersuchung 
Die Ergebnisse des t-Tests zeigen wieder, dass es keine statistisch signifikanten Unterschiede in den Leistungen der Gruppen gab, da der Wert $\mathrm{p}>$ 0,05 ist.

\subsection{Ergebnisse der Posttests}

Test 1

$\mathrm{Zu}$ Beginn der Untersuchung wurde mit beiden Gruppen ein standardisierter Test der Stufe A2 durchgeführt. Da beide Gruppen diesen Test relativ gut bestanden, und keine statistisch relevanten Unterschiede zwischen den Gruppen festgestellt wurden, wurde mit den Lernenden am Ende der Untersuchung der Test für das Goethe-Zertifikat auf Stufe B1 durchgeführt (Balser et al. 2014). Die Ergebnisse dieses Tests sind in Tabelle 4 dargestellt.

\begin{tabular}{|c|c|c|c|c|c|c|c|}
\hline TEST 1 & Gruppe & $N$ & $M W$ & $S A$ & Unterschied im MW & $t$ & $p$ \\
\hline \multirow{2}{*}{ T1 Hören } & $E G$ & 17 & 22,29 & 3,37 & \multirow{2}{*}{1,18} & \multirow{2}{*}{1,032} & \multirow{2}{*}{0,310} \\
\hline & $K G$ & 17 & 21,12 & 3,28 & & & \\
\hline \multirow{2}{*}{ T1 Lesen } & $E G$ & 17 & 15,88 & 3,28 & \multirow{2}{*}{2,00} & \multirow{2}{*}{1,467} & \multirow{2}{*}{0,152} \\
\hline & $K G$ & 17 & 13,88 & 4,57 & & & \\
\hline \multirow{2}{*}{$\begin{array}{l}\text { T1 } \\
\text { Schreiben }\end{array}$} & $E G$ & 17 & 68,76 & 26,12 & \multirow{2}{*}{28,59} & \multirow{2}{*}{2,897} & \multirow{2}{*}{0,007} \\
\hline & $K G$ & 17 & 40,18 & 31,21 & & & \\
\hline
\end{tabular}

Tab. 4. Ergebnisse von Test 1 nach Durchführung der Untersuchung

Die Tabelle zeigt, dass statistisch signifikante Unterschiede nur im Bereich des Schreibens festgestellt wurden $(t=2,897, p=0,007)$.

Test 2

Mit diesem nicht standardisierten Test wurde die Lesekompetenz anhand eines wieder leicht gekürzten und vereinfachten authentischen Textes (Süddeutsche Zeitung 2011) überprüft. Zudem wurde die Schreibkompetenz geprüft, indem die Lernenden ihre Meinung zum Artikel äußerten und noch zwei Aufsätze verfassten. Die Ergebnisse dieses Tests sind in Tabelle 5 dargestellt. 


\begin{tabular}{|c|c|c|c|c|c|c|c|}
\hline TEST 2 & Gruppe & $N$ & $M W$ & $S A$ & Unterschied im $M W$ & $t$ & $p$ \\
\hline \multirow{2}{*}{ T2 LESEN } & $E G$ & 16 & 10,75 & 1,49 & \multirow{2}{*}{1,16} & \multirow{2}{*}{1,570} & \multirow{2}{*}{0,127} \\
\hline & $K G$ & 16 & 9,59 & 2,54 & & & \\
\hline \multirow{2}{*}{ T2 SCHREIBEN } & $E G$ & 16 & 19,88 & 7,58 & \multirow{2}{*}{5,31} & \multirow{2}{*}{2,133} & \multirow{2}{*}{0,041} \\
\hline & $K G$ & 16 & 14,56 & 6,46 & & & \\
\hline
\end{tabular}

Tab. 5. Ergebnisse von Test 2 nach Durchführung der Untersuchung

Im zweiten Test wurden nur im Bereich des Schreibens signifikante Unterschiede festgestellt $(\mathrm{t}=2,133 ; \mathrm{p}=0,041)$, während der Lesetest keine Unterschiede ergab.

Test 3

Mit diesem nicht standardisierten Test wurde die Hörkompetenz anhand einer anderen Folge der Fernsehserie Mein Leben \& Ich überprüft. Auch die Schreibkompetenz wurde getestet, indem die Lernenden die gezeigte Folge mit eigenen Worten zusammenfassen sollten.

\begin{tabular}{|c|c|c|c|c|c|c|c|}
\hline TEST 3 & Gruppe & $N$ & $M W$ & $S A$ & Unterschiede im $M W$ & $t$ & $p$ \\
\hline \multirow{2}{*}{ T3 Hören } & $E G$ & 17 & 8,50 & 1,87 & \multirow{2}{*}{0,24} & \multirow{2}{*}{0,371} & \multirow{2}{*}{0,713} \\
\hline & $K G$ & 17 & 8,26 & 1,83 & & & \\
\hline \multirow{2}{*}{ T3 Schreiben } & $E G$ & 17 & 10,32 & 3,23 & \multirow{2}{*}{4,24} & \multirow{2}{*}{3,868} & \multirow{2}{*}{0,001} \\
\hline & $K G$ & 17 & 6,09 & 3,16 & & & \\
\hline
\end{tabular}

Tab. 6. Ergebnisse von Test 3 nach Durchführung der Untersuchung

In Test 3 wurden nur im Bereich des Schreibens statistisch signifikante Unterschiede festgestellt $(t=3,868 ; p=0,001)$, wie bei den beiden anderen Tests.

\subsection{Fragebögen}

Neben den Tests wurde den Lernenden auch ein Fragebogen ausgehändigt, in dem sie ihre Eindrücke und Erfahrungen mit diesem Sprachförderprogramm äußern sollten. Zunächst sollten die Lernenden der Experimentalgruppe auf der Skala des semantischen Differentials (1-7) angeben, wie effektiv sie die Arbeit mit authentischen Texten einschätzen. Dabei steht 1 für ,nicht effektiv“ und 7 für ,sehr effektiv“, während 4 für eine neutrale Haltung steht. Die Ergebnisse sind in Tabelle 7 aufgezeigt. 


\begin{tabular}{|c|c|c|c|c|}
\hline $\begin{array}{l}\text { Wie schätzen Sie die Effektivität des Einsatzes von } \\
\text { authentischen Texten ein? }\end{array}$ & $N$ & $\%$ & $M W$ & $S A$ \\
\hline 4 (neutrale Haltung, nicht effektiv aber auch nicht ineffektiv) & 2 & 11,1 & \multirow[t]{4}{*}{6,44} & \multirow[t]{4}{*}{0,98} \\
\hline 6 (ziemlich effektiv) & 4 & 22,2 & & \\
\hline 7 (sehr effektiv) & 12 & 66,7 & & \\
\hline Insgesamt & 18 & 100,0 & & \\
\hline
\end{tabular}

Tab. 7. Einschätzung der Effektivität von authentischen Texten in der Experimentalgruppe am Ende der Untersuchung

Die Tabelle zeigt einen hohen Durchschnittswert für diese Frage (MW = 6,44, SA = 0,98), was bedeutet, dass die Teilnehmer der Experimentalgruppe den Einsatz von authentischen Texten im Unterricht als sehr effektiv einschätzen (Stipančević 2018).

Im Rahmen der nächsten Frage sollten Lernende ihre Bewertung aus der ersten Frage begründen. Diese Erklärungen wurden gruppiert und sind in der Tabelle 8 aufgeführt:

\begin{tabular}{lll}
\hline & Erklärungen der Lernenden der Experimentalgruppe & $\boldsymbol{N}$ \\
\hline 1 & Großer Fortschritt in Sprachkenntnissen & 8 \\
\hline 2 & Interessantere Art des Lernens & 5 \\
\hline 3 & Ich spreche leichter und verstehe besser & 4 \\
\hline 4 & Ich finde diese Art des Lernens schwieriger & 1 \\
\hline 5 & Ich habe keine Fortschritte bemerkt & 1 \\
\hline
\end{tabular}

Tab. 8. Erklärungen der Lernenden der Experimentalgruppe für ihre Bewertung aus der vorherigen Frage

Die ersten drei Äußerungen stammen von den Lernenden, die diesen Ansatz auf der Skala des semantischen Differentials hoch bewertet haben (mit Zahlen 6 und 7), während die letzten zwei Äußerungen von den Lernenden stammen, die den extensiven Ansatz nicht besonders effektiv aber auch nicht ineffektiv bewertet haben (mit der Zahl 4).

\section{DISKUSSION DER ERGEBNISSE}

Das Ziel dieser Untersuchung war es, festzustellen, welche Effekte der extensive Ansatz auf die Sprachkompetenz der Lernenden hat. Man ging davon aus, dass sich die größten Unterschiede im rezeptiven Bereich feststellen lassen. Die Ergebnisse der Untersuchung zeigen jedoch, dass die signifikanten Unterschiede zwischen den Gruppen den produktiven Bereich betrafen. Die 
Ergebnisse dieser Untersuchung waren überraschend, denn von den meisten Forschern wird hervorgehoben, dass sich durch den extensiven Ansatz vor allem die rezeptiven Kompetenzen verbessern (vgl. Edmondson 1996; Elley 1991; Elley \& Mangubhai 1981, 1983; Ellis 1994). Davon ging auch Biebricher (2008) in ihrer experimentellen Untersuchung aus, in der sie sich mit den Effekten des extensiven Lesens auf die Lesekompetenz im Englischunterricht beschäftigte. Die gesamte Untersuchung erstreckte sich über einen Zeitraum von vier Monaten und die Autorin konnte feststellen, dass keine signifikanten Unterschiede in den Leistungen der Experimental- und Kontrollgruppe bestanden, obwohl die Experimentalgruppe einem wesentlich stärkeren Input ausgesetzt war. Andererseits wurde festgestellt, dass im Test, mit dem die allgemeine Sprachkompetenz gemessen wurde, signifikante Unterschiede in den Leistungen zwischen der Experimental- und Kontrollgruppe vorlagen.

Die Ergebnisse dieser Untersuchung weisen viele Ähnlichkeiten mit den Ergebnissen der Untersuchung auf, die in diesem Artikel beschrieben wurde. Die Experimentalgruppe schnitt nach Ende der Untersuchung doch leicht besser im Hör- und Leseverstehenstest ab als die Kontrollgruppe, es wurden jedoch keine statistisch signifikanten Unterschiede zwischen den Gruppen festgestellt. Im Gegensatz dazu ließen sich statistisch signifikante Unterschiede zwischen den Gruppen in allen drei Schreibtests feststellen, zugunsten der Experimentalgruppe. Leider wurde im Rahmen dieser Untersuchung nicht die mündliche Sprachproduktion der Lernenden getestet, da dies viel zu anspruchsvoll gewesen wäre. Das könnte aber eine der Aufgaben und Ziele der nächsten Untersuchungen sein.

\section{FAZIT}

Die Vorteile des extensiven Ansatzes für den Fremdsprachenunterricht sind bemerkenswert, weshalb er ein integraler Bestandteil des Unterrichts werden sollte. Durch einen verstärkten Input und eine reichhaltige Lernumgebung kann die Sprache nicht nur bewusst, sondern auch unbewusst erworben werden, was die Effizienz des gesamten Spracherwerbsprozesses steigern kann.

Zur richtigen Umsetzung des extensiven Ansatzes ist es wichtig, den Lernenden die Freiheit zu geben, Texte zu lesen/hören, die sie persönlich interessieren, da sich dies positiv auf ihre Motivation auswirkt. Außerdem ist noch wichtig, dass authentische Texte gelesen/gehört werden, da solche Texte die Lernenden motivieren und ihr Interesse wecken. Dabei sollten sowohl Lese- als auch Hörtexte einbezogen werden, denn beide Kompetenzen stellen eine wichtige 
Voraussetzung zur Entwicklung der produktiven Fertigkeiten dar. Damit die Lernenden tatsächlich Fortschritte machen können, ist zudem wichtig, dass das Lesen und Hören immer mit schriftlichen und mündlichen Aktivitäten verbunden wird, da dies laut Output-Hypothese den Spracherwerbsprozess besonders vorantreibt. Wenn man all diese Methoden und Techniken in den Unterricht einbaut, wird man schon nach einem Semester feststellen können, was für Fortschritte die Lerngruppe in Hinblick auf ihre Sprachkompetenzen gemacht hat. Zudem wird man noch zufriedeneren und motivierteren Lernende begegnen, die mit viel Vergnügen, und Interesse dem Deutschlernen nachgehen.

\section{LITERATUR}

Aufderstraße, H., Müller, J., Storz, Th. (2003). Delfin. A2. Lehr- und Arbeitsbuch. Ismaning: Hueber.

Balser, A., Glotz-Kastanis, J., Papadopoulou, M., Paradi-Stai, D., Vosswinkel, A. (2014). Zertifikat B1 neu. 15 Übungsprüfungen. München: Hueber.

Barnett, M. A. (1989). More than meets the eye: Foreign language learner reading. Theory and practice. Englewood Cliffs, NJ: Prentice Hall Regents.

Biebricher, Ch. (2008). Lesen in der Fremdsprache. Eine Studie zu Effekten extensiven Lesens. Tübingen: Gunter Narr.

Biederstädt, W. (1990). Die Schulung des extensiven Lesens im Englischunterricht der Realschule - Anregungen zur Förderung einer vernachlässigten Fertigkeit. Die Realschule 98 (7): 285-288.

Börner, O. \& Brusch, W. (2004). Die Hamburger Bücherkiste für den Englischunterricht der Grundschule. Englisch. Zeitschrift für Englischlehrerinnen und Englischlehrer 4 (4): 146-152.

Brusch, W. (1994). Erziehung zum Lesen im Englischen durch Klassenbibliotheken. Ein empirisches Unterrichtsprojekt. Praxis des neusprachlichen Unterrichts 41 (1): 17-26.

Burwitz-Melzer, E. (2004). Growing Up Literally - Authentische Bilderbücher und ihre Erarbeitung im frühen Fremdsprachenunterricht. In Literaturdidaktik im Dialog, Hrsg. L. Bredella, W. Delanoy, \& C. Surkamp (Tübingen: Gunter Narr): 123-146.

Day, R. \& Bamford, J. (1998). Extensive Reading in the Second Language Classroom. Cambridge: Cambridge University Press.

Decke-Cornill, H. \& Küster, L. (2014). Fremdsprachendidaktik. Eine Einführung. Tübingen: Narr Francke. 
Düwell, H. (1979). Fremdsprachenunterricht im Schülerurteil. Untersuchungen zu Motivation, Einstellungen und Interessen von Schülern im Fremdsprachenunterricht Schwerpunkt Französisch. Tübingen: Gunter Narr Verlag.

Edmondson, W. J. (1996). Textverstehensprozesse/Spracherwerbsprozesse. In Texte im Fremdsprachenerwerb: verstehen und produzieren, Hrsg. W. Börner, \& K. Vogel (Tübingen: Narr): 107-121.

Elley, W. (1991). Acquiring Literacy in a Second Language: The Effect of book-based programs. Language Learning, 41(3): 375-411.

Elley, W. \& Mangubhai, F. (1981). The Impact of a Book Flood in Fiji Primary Schools. Wellington: New Zealand Council for Educational Research.

Ellis, N. (1994). Consciousness in Second Language Learning: Psychological Perspectives on the Role of Conscious Processes in Vocabulary Acquisition. Aila Review 11: 37-56.

Estes, T. H., \& Vaughan, J. L. (1973). Reading interest and comprehension. Implications. The Reading Teacher 27: 149-153.

Gerbes, J., \& van der Werff, F. (2007). Fit fürs Goethe-Zertifikat A2. Start Deutsch 2. Ismaning: Hueber.

Hilpert, S., Niebisch, D., Penning-Hiemstra, S., Specht, F., Reimann, M. \& Tomaszewski, A. (2006). Schritte international 3. Lehr- und Arbeitsbuch. Ismaning: Hueber.

Hilpert, S., Kerner, M., Niebisch, D., Specht, F., Weers, D., Reimann, M., Tomaszewski, A. (2007). Schritte international 4. Lehr- und Arbeitsbuch. Ismaning: Hueber.

Hulstijn, J. H. (1992). Retention of inferred and given word meanings: experiments in incidental vocabulary learning, In Vocabulary and Applied Linguistics, Hrsg. P. Arnaud \& H. Béjoint (London: Macmillan Academic and Professional): $113-125$.

Koeppel, R. (2013). Deutsch als Fremdsprache - Spracherwerblich reflektierte Unterrichtspraxis. Baltmannsweiler: Hohengehren.

Krashen, S. D. (1985). The Input Hypothesis. Issues and Implication. London, New York: Addison-Wesley Longman.

Müller, M., Rusch, P., Scherling, T., Schmitz, H., Wertenschlag, L. (2011). Optimal B1. Lehr- und Arbeitsbuch. Berlin: Klett.

Nation, I. S. P. (2001). Learing Vocabulary in Another Language. Cambridge: Cambridge University Press.

Paribakht, T. S., \& Wesche, M. (1997). Vocabulary enhancement activities and reading for meaning in second language vocabulary acquisition. In Second Language 
Vocabulary Acqusition, Hrsg. J. Coady \& T. Huckin (Cambridge: Cambridge University Press): 174-200.

Ross, I., \& Porteous-Schwier, G. (2005). Readers in class. What's new? Das Englisch Magazin: 7-9.

Seow, A. (1999). What do we really want out of USSR? Teaching of English Language and Literature (TELL) Journal 15 (2): 22-24.

Silva, J. (1992). Extensive Reading: No Pain, No Gain? English Teaching Forum 30 (2): 48-49.

Stenzel, K. (1990). Die Klassenbibliothek als ein Modell für das extensive Lesen. Praxis des neusprachlichen Unterrichts 27 (4): 361-364.

Stenzel, K. (1994). Lesetraining mit der englischen Klassenbibliothek. Training des extensiven Lesens. Schulmagazin 5-10, 9 (3): 29-32.

Stipančević, A. (2013). Medien im DaF-Unterricht in Serbien, In Treffpunkte Literatur, Sprache und Didaktik im deutsch-serbischen Dialog, Hrsg. J. BeliGöncz, I. Boose, B. Petronijević und A. Wrobel (Ludwigsburg: Schneider Verlag Hohengehren): 264-280.

Stipančević, A. (2014). Die Vermittlung der interkulturellen Kompetenz im Unterricht Deutsch als Fremdsprache durch die Rezeption von Fernsehserien, In Fremdsprachenunterricht im Spannungsfeld zwischen Sprachwissen und Sprachkönnen. Saabrücker Schriften zu Linguistik und Fremdsprachendidaktik (SSLF), Hrsg. Th. Tinnefeld (Saarbrücken: htw saar): 491-501.

Stipančević, A. (2015). Motivacija za učenje nemačkog jezika kao izbornog predmeta na Filozofskom fakultetu. In Strani jezici na Filozofskom fakultetu: primenjenolingvistička istraživanja, ur. B. Radić-Bojanić (Novi Sad: Filozofski fakultet): 69-90.

Stipančević, A. (2016). Efekti primene autentičnih tekstova u nastavi nemačkog jezika na veštinu pisanja i znanja vokabulara. Nastava $i$ vaspitanje 3: 613-627.

Stipančević, A. (2018). Uloga i značaj autentičnih tekstova u nastavi nemačkog jezika - rezultati kvalitativnog istraživanja. Zbornik radova Filozofskog fakulteta Univerziteta u Prištini 48 (1): 43-58.

Storch, G. (2009). Deutsch als Fremdsprache. Eine Didaktik. Paderborn: Fink.

Surkamp, C. (2010). Metzler Lexikon. Fremdsprachendidaktik. Stuttgat, Weimar: Metzler Verlag.

Swerlova, O. (2013). Grammatik und Konversation 1. München: Klett-Langenscheidt. Volkmann, L. (2000). Unterhaltungsliteratur - Schulerinnen und Schüler beim Lesen begleiten. Der fremdsprachliche Unterricht Englisch 6: 4-11. 
Weyers, J. R. (1999). The Effect of Authentic Video on Communicative Competence. The Modern Language Journal 83 (3): 339-349.

Wolff, D. (1997). Lehren lernen. Wege zur Autonomie des Schülers. Lernmethoden Lehrmethoden. Wege zu Selbständigkeit. Friedrich Jahresheft 15: 106-108.

Ana I. Stipančević

Univerzitet u Novom Sadu

Filozofski fakultet, Odsek za germanistiku

\section{PODSTICANJE JEZIČKIH KOMPETENCIJA KROZ EKSTENZIVNO ČITANJE I SLUŠANJE U UNIVERZITETSKOJ NASTAVI NEMAČKOG JEZIKA}

\section{Sažetak}

O ekstenzivnom čitanju postoje mnogobrojna istraživanja, koja su sprovedena, pre svega, u kontekstu nastave engleskog jezika, dok su istraživanja koja se bave ekstenzivnim čitanjem u kontekstu nastave nemačkog jezika izuzetno retka. Osim toga, istraživanja koja se bave ekstenzivnim slušanjem su posebno zapostavljena, te im je neophodno posvetiti više pažnje u naučnoj i stručnoj literaturi. U ovom radu su prikazani rezultati empirijskog istraživanja koje je sprovedeno 2014/15. godine na Filozofskom fakultetu sa studentima koji uče nemački kao izborni jezik, nivoa znanja A2. Dok je u eksperimentalnoj grupi primenjen ekstenzivan pristup, u kontrolnoj grupi nastava se odvijala na tradicionalan način uz udžbenike i druge didaktičke materijale. Istraživanje je trajalo dva semestra, nakon čega su obe grupe testirane. Rezultati su statistički obrađeni, dok su razlike u rezultatima između grupa utvrđene t-testom. Rezultati istraživanja ukazuju da ekstenzivni programi čitanja i slušanja pozitivno utiču na produktivne jezičke veštine, ukoliko se tekstovi, koji se slušaju i čitaju, obogate odgovarajućim didaktičkim postupcima, tako da primena znanja jezika bude u prvom planu. Da bi se na pravilan način sproveo ekstenzivan pristup, važno je učenicima dati slobodu u izboru tekstova za slušanje i čitanje, pri čemu ih treba upućivati na upotrebu autentičnih sadržaja i korišćenje internet-izvora, budući da je takav način rada i učenja blizak učeničkoj supkulturi, razvija kod njih osećaj samostalnosti i sigurnosti, kao i svest o primeni znanja jezika, što predstavlja važan motivacioni faktor u nastavi stranog jezika.

Ključne reči: ekstenzivno čitanje i slušanje, eksperimentalno istraživanje, autentični tekstovi, nastava nemačkog jezika.

Eingegangen: 5. 4. 2020.

Annahme: 10. 8. 2020. 